\section{Minicentro comercial}

Andrés Mas, Cristobal Fernández Vitacura, Chile
Una sociedad más compleja deviene en la existencia de corrientes paralelas, opuestas o complementarias. El mercado chileno interno, antes teñido por la omnipresente figura del mall, da lugar boy a otros modelos de urbanidad y consumo completando una gama de escalas que supera la polaridad megamercado v/s kiosko.

Palabras clave: Arquitectura-Chile, espacios comerciales, tienda, retail,
A complex society allows the existence of parallel, opposite or complementary trends. The internal Chilean marketplace, utterly influenced by the image of the mall, lately is opening to alternative models of urbanity and consumption. These models complete a wide range of scales, between the bypermarket and the kiosk.

Key words: Architecture-Chile, commercial buildings, store, retai

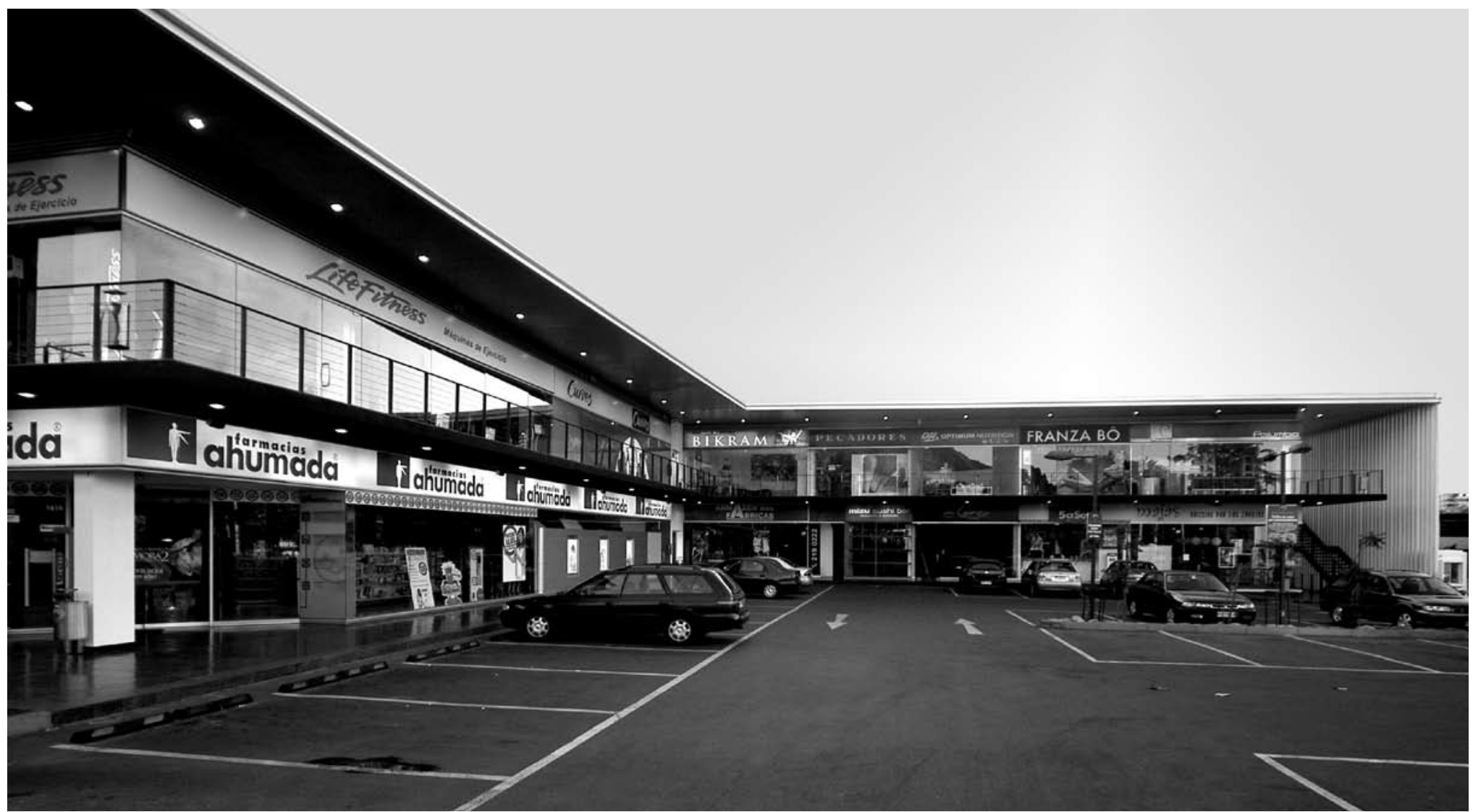

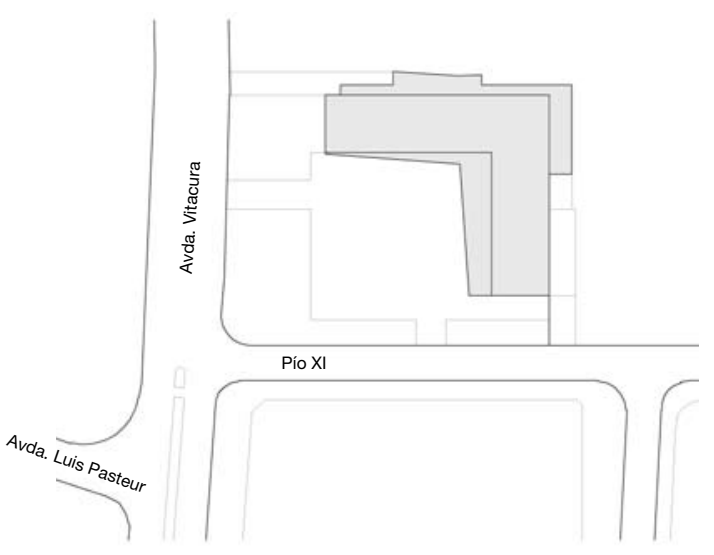

Planta emplazamiento E/1:2.000
Una arquitectura al servicio de la comunidad / Abiertos todos los días del año, los grandes centros comerciales -los llamados palacios del consumo- cambiaron durante la última década los hábitos de la sociedad chilena. En ellos no sólo se compra, también se pasea, se conversa y se come; todo bajo un mismo espacio cerrado, sin insertarse en el medio que lo rodea. Una verdadera ciudad techada. Sin embargo, últimamente se ha visto el surgimiento de un nuevo tipo comercial en Santiago, que en algo nos recuerda aquella época de nuestros abuelos, cuando se compraba en el almacén de la esquina, donde las más de las veces el cliente conocía el nombre del dueño que era el mismo que los atendía.

En EE.UU. se les denomina strip center y aquí les llaman minicentro comercial. Si bien el concepto de este comercio vecinal es importado, la aceptación de nuestra sociedad fue inmediata ya que integra lo mínimo necesario para garantizar los servicios básicos de la comunidad a la que sirve: panadería, farmacia, gimnasio, café y cómodos estacionamientos. De esta forma, se rescata algo de la añorada vida de barrio.

La mayoría de estas nuevas plazas comerciales liberan la esquina para generar un edificio de hormigón de dos pisos en forma de $L$, que busca orientar los locales y mostrarse en su totalidad hacia la calle en forma transparente.

El modelo de minicentro comercial, que resuelve una nueva línea de edificación para terrenos en esquinas que forman parte de avenidas principales, entrega una propuesta arquitectónica con valor, que ha logrado controlar la publicidad que juega en contra de casi todos los proyectos comerciales. El edificio se defiende: se logran ver las cuatro esquinas trabajadas del mismo modo, presentando una gran amplitud, algo que incide fuertemente en el agrado de un barrio. Lo anterior es lo que hemos querido ver reflejado en la esquina ubicada en Avenida Vitacura con Pío XI: sin pretensiones ni grandilocuencia, esta propuesta arquitectónica comercial responde de una manera equilibrada a un barrio de la ciudad. ARQ 

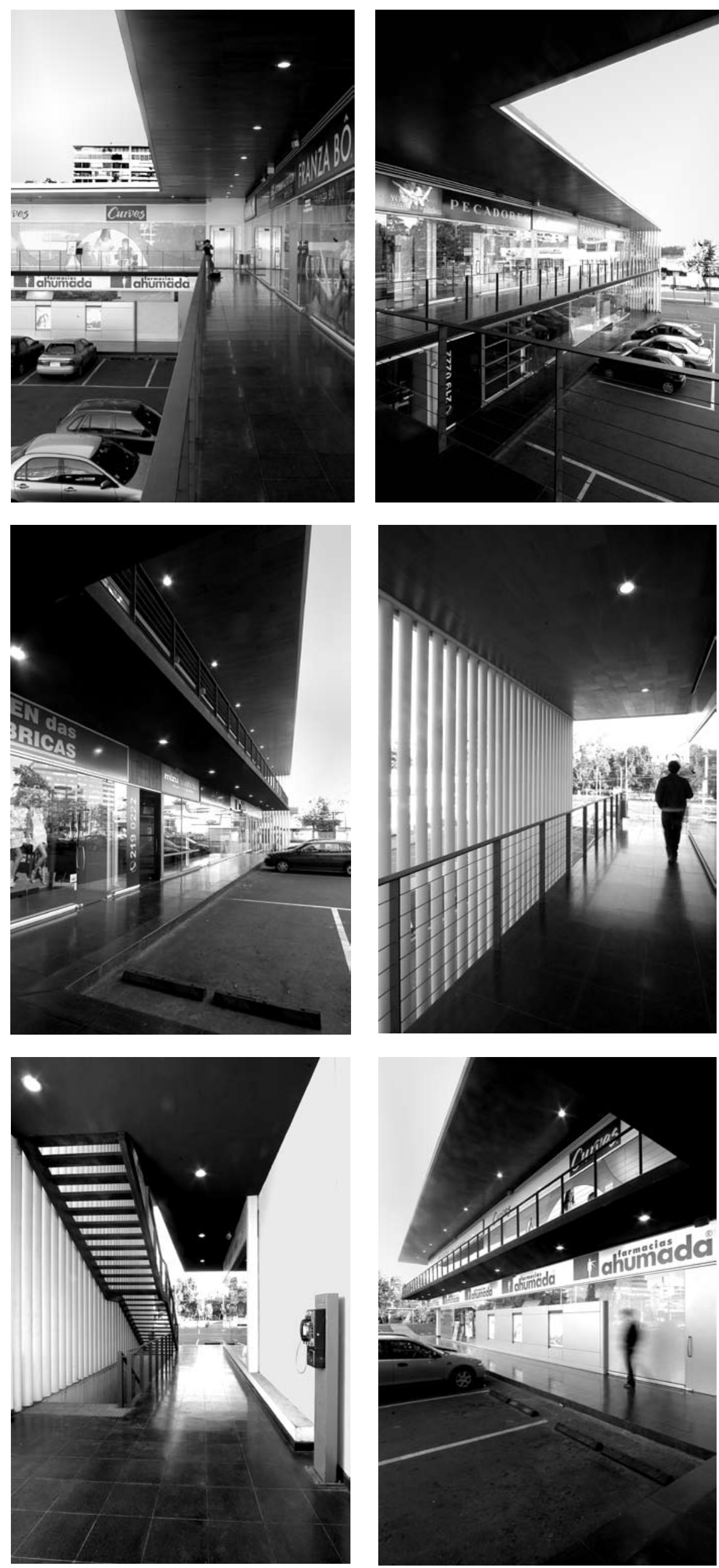

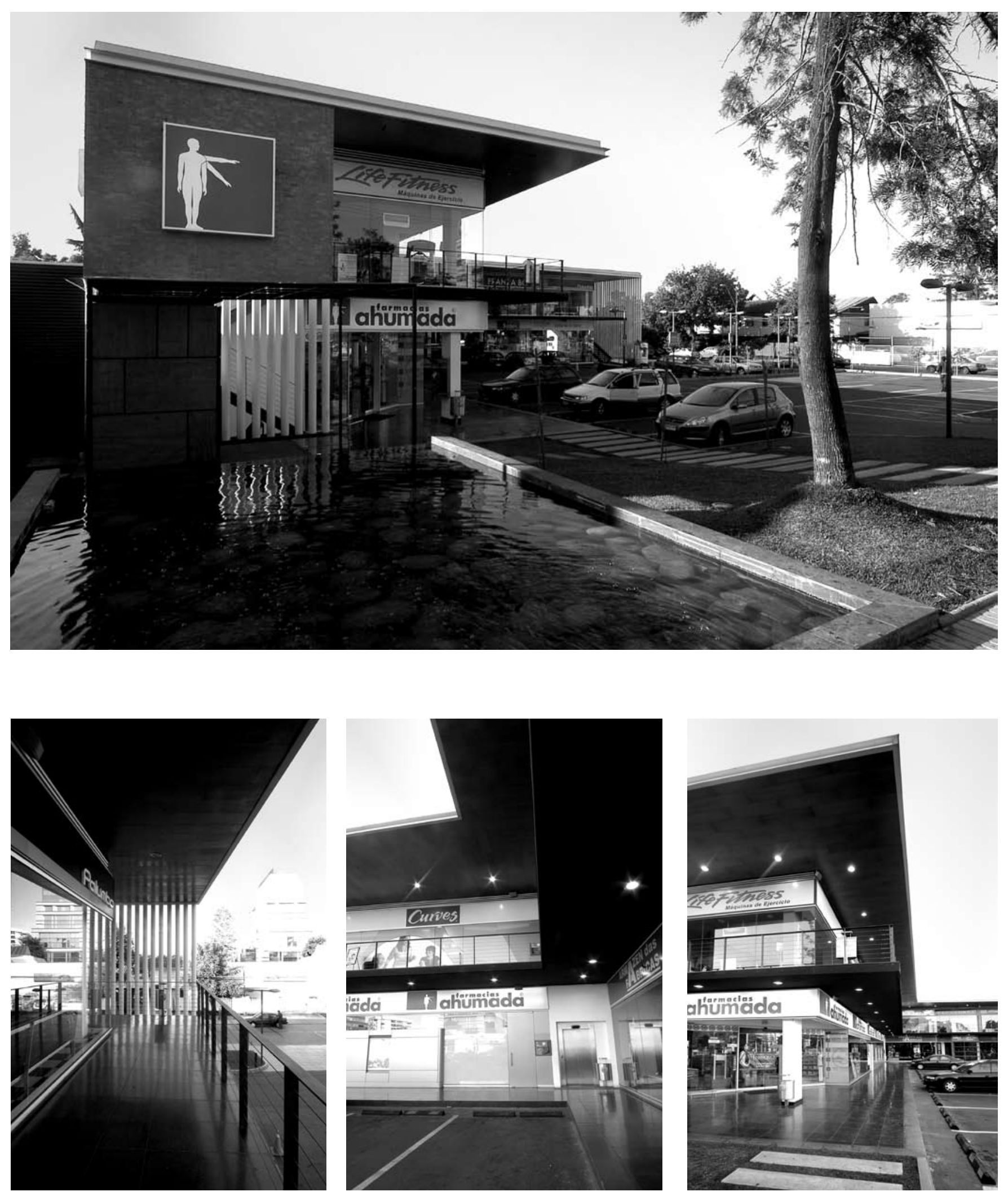


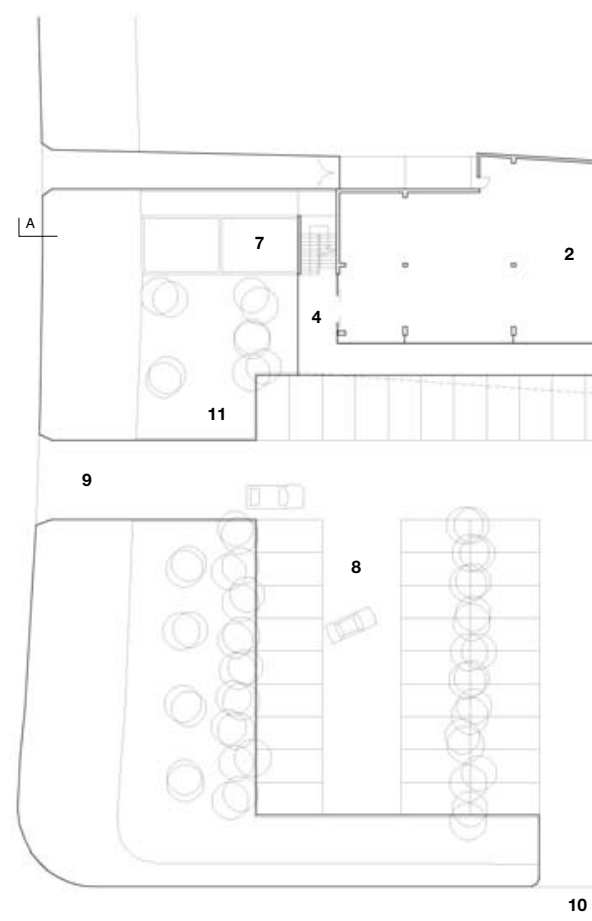

Planta primer piso E/1:750
10

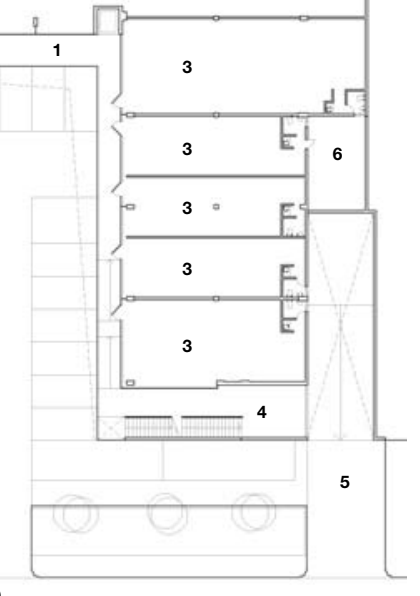

1 Corredor exterior 2 Sala de ventas principal 3 Local comercial

4 Atrio circulación vertical 5 Acceso vehicular servicios 7 Pileta

8 Estacionamientos

9 Acceso estacionamientos

Vitacura

10 Acceso es

11

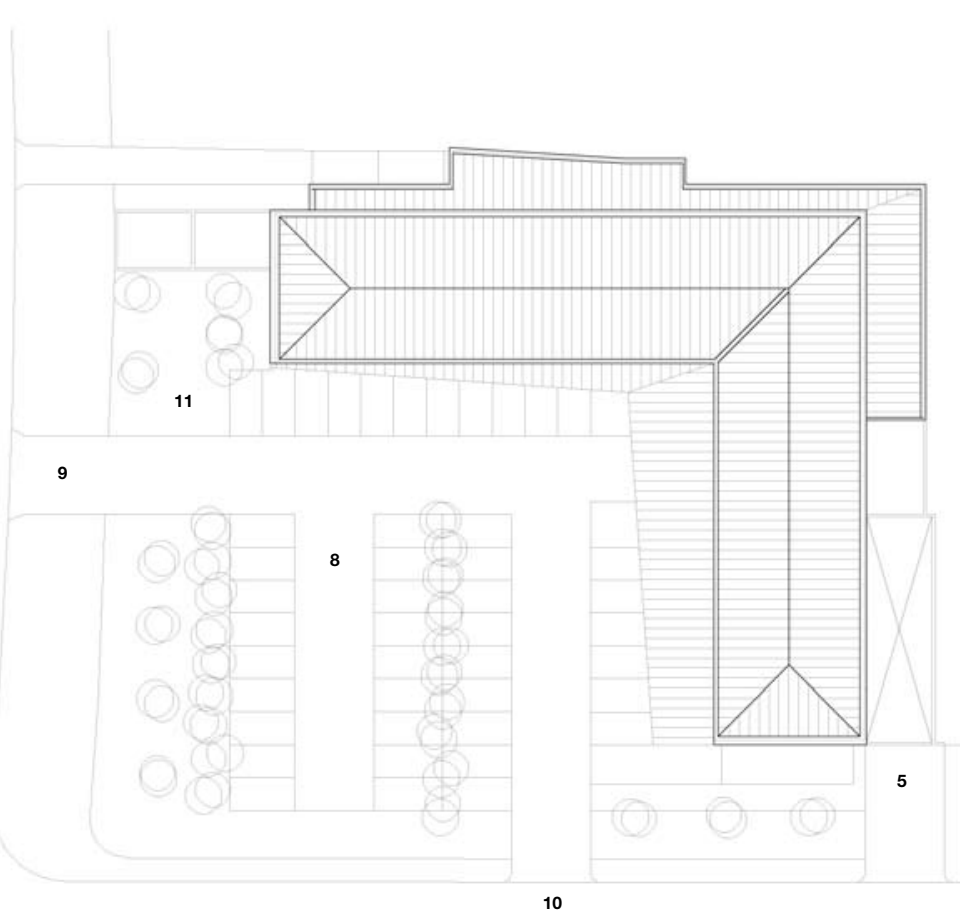

Planta cubiertas
ARQ

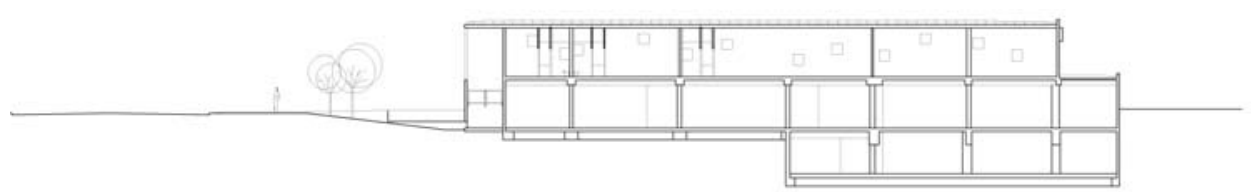

Corte AA

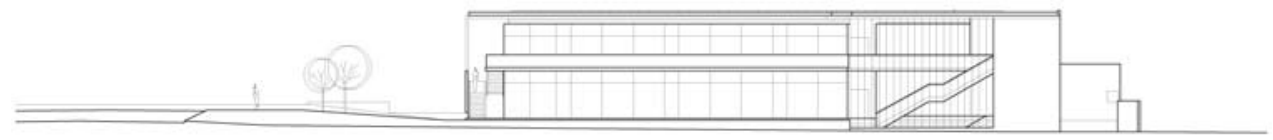

Elevación poniente
Minicentro comercial

Arquitectos Andrés Mas, Cristóbal Fernández

Arquitecto colaborador Rodrigo Santa María

Ubicación Pío XI esq. Avda. Vitacura, Vitacura, Chile

Cliente Administraciones y Rentas S.A.

Cálculo estructural Patricio Stagno

Construcción Constructora San Gerardo

Inspección técnica Mas Fernández arquitectos

Instalaciones sanitarias Hidrosur Ltda.

Proyecto eléctrico Mauricio López - Ingemel Ltda.

Materialidad hormigón armado, estructura metálica de

techumbre, cubierta de membrana asfáltica, cierros de

carpintería de aluminio y vidrio, cielos de terciado

marino de cedro, pavimentos de baldosa microvibrada

Presupuesto $15 \mathrm{UF} / \mathrm{m}^{2}\left(\mathrm{US} \$ 466 / \mathrm{m}^{2}\right)$

Superficie terreno $2.990 \mathrm{~m}^{2}$

Superficie construida $2.595 \mathrm{~m}^{2}$

Año proyecto 2003

Año construcción 2004

Fotografía Álvaro Benítez 